\title{
Effect of Addition and Molecular Size of Triglyceride Oils on Phase Behavior and Surfactant Self-Assemblies
}

\author{
Kenji Aramaki* and Hossain Md. KhaLID \\ Graduate School of Environment and Information Sciences, Yokohama National University \\ (Tokiwadai 79-7, Hodogaya-ku, Yokohama 240-8501)
}

Edited by T. Kato, Tokyo Metr. Univ., and accepted June 22, 2004 (received for review May 13, 2004)

\begin{abstract}
The cloud temperature of $\mathrm{C}_{12} \mathrm{EO}_{8}$ aqueous solution decreases by solubilizing triglyceride oils (1,2,3-trihexanoylglycerol (THG), 1,2,3-trioctanoylglycerol (TOG) and 1,2,3tridecanoylglycerol (TDG)). The reduction of cloud temperature is larger with the oil having shorter alkyl chains. In the water $/ \mathrm{C}_{12} \mathrm{EO}_{8}$ system, a micellar, a hexagonal, a bicontinuous cubic and a lamellar phases are successively formed with increasing surfactant concentration at $25^{\circ} \mathrm{C}$. A discontinuous cubic phase is formed by adding TOG to the aqueous $\mathrm{C}_{12} \mathrm{EO}_{8}$ system. SAXS measurement was performed on the discontinuous cubic, hexagonal and lamellar phases and molecular parameters such as effective cross sectional area per surfactant molecule, radius of micelle for the discontinuous cubic and the hexagonal phases, half-thickness of bilayer of the lamellar phase were obtained. The changes in the molecular parameters by increasing TOG content suggest that most of the oil molecules are solubilized in the core of surfactant aggregates. Thermal stability of the discontinuous cubic phase increases. The amount of solubilized oil in the discontinuous cubic phase increases by increasing temperature and decreasing oil molecular weight.
\end{abstract}

Key words: triglyceride oil, oil solubilization, cubic phase, phase behavior, small angle Xray scattering

\section{Introduction}

Triglyceride oil (1,2,3-trialkanolyglycerol) is a main ingredient of food oils. Many food products are in a form of emulsion that mainly contains water, oil and emulsifier (normally surfactant). Emulsions can be categorized in two types, oil-in-water $(\mathrm{O} / \mathrm{W})$ or water-inoil (W/O), which normally depends on the hydrophilelipophile balance (HLB) of surfactant used. However the emulsion type is also changed by the property of oil (1) even if the surfactant HLB is fixed.

Emulsions are normally formulated by shaking a sample of a multi-phase equilibrium, which is normally micellar phase and excess oil phase, or reverse micellar phase and excess water phase. It is also possible to formulate emulsions in another multi-phase sample containing liquid crystal. It is reported that stable $\mathrm{O} / \mathrm{W}$ or $\mathrm{W} / \mathrm{O}$ emulsions can be formulated with utilizing lamellar or reverse hexagonal phase $(2,3)$. Since dispersed phase is covered by liquid crystals, stability of the obtained emulsion is high. Kunieda et al. $(4,5)$ reported that the emulsion formulated from the two-phase equilibrium of a discontinuous cubic phase and an oil phase is highly viscous and transparent due to the high viscosity of the continuous phase being the cubic phase and the contrast matching of refractive indices of the continuous phase (cubic phase) and the dispersed phase (oil). They also reported the gel-emulsion in which water

\footnotetext{
* Correspondence to: Kenji ARAmaKI, Graduate School of Environment and Information Sciences, Yokohama National University, Tokiwadai 79-7, Hodogaya-ku, Yokohama 240-8501

E-mail: aramakik@ynu.ac.jp
} 
droplets are dispersed in a matrix of a reverse-discontinuous cubic phase (6).

Cubic phase is a gel-like phase with high viscosity and transparency. It is categorized in four types, discontinuous type, bicontinuous type, reverse bicontinuous type and reverse discontinuous type. Discontinuousand reverse-discontinuous-cubic phase contain spherical micelles with positive and negative curvature, respectively. The cubic-phase-stabilized emulsion mentioned in the previous paragraph has a continuous phase being the discontinuous or a reverse discontinuous cubic phase. The discontinuous-type cubic phase is normally formed between a micellar phase and a hexagonal phase in a phase diagram as a function of concentration of surfactant. Since spherical micelles have to maintain their structure at high surfactant concentration to form a discontinuous cubic phase, it is normally formed in aqueous surfactant systems with very hydrophilic nonionic surfactants, e.g. poly(oxyethylene)-type surfactant (7), poly(oxyethylene)-poly(oxypropylene)-poly (oxyethylene) triblock copolymer (8), polyglycerol ester (9), sucrose ester (10). However adding oil to a micellar phase or a hexagonal phase in an aqueous surfactant system often induces a formation of a discontinuous cubic phase $(5,11-13)$ since a structural transition of rod-like or elongated micelles to spherical micelles often takes place by the solubilization of oil (14). The amount of adding oil needed for the formation of discontinuous cubic phase increases with decreasing molecular size of hydrocarbons and decreasing surfactant hydrophilicity (12).

If the oil molecules are solubilized in the core of the self-assemblies, a positive surfactant layer curvature increases (1) or a zero curvature is unchanged (11). On the other hand if the oil molecules penetrate into a surfactant palisade layer, a positive curvature becomes less positive or even it is turned into a negative curvature (1). Note that a positive and a negative curvature of surfactant layer indicate a surfactant layer that is convex toward water and oil, respectively. The curvature of normal-type micelle or hexagonal phase tends to be less positive by the solubilization of oils with penetration tendency and to be more positive by the solubilization of oils with less-penetration tendency $(1,11)$. Hence the phase transition from a micellar phase or a hexagonal phase to a discontinuous cubic phase takes place in the case of adding oils having less penetration tendency (13). In previous reports $(1,11)$, hydrocarbons or aro- matic oils are mainly used to discuss the relation between the solubilization site of oils and the structural change of self-assemblies. However there are few reports for triglyceride oils in spite of the importance of practical applications.

In this paper we report the phase behavior of water/poly(oxyethylene)-type nonionic surfactant/ triglyceride oil systems to evaluate the effect of addition and molecular size of triglyceride oils on the structure of surfactant self-assemblies.

\section{Experimental}

\subsection{Materials}

Homogenous octa(oxyethylene) dodecyl ether $\left(\mathrm{C}_{12} \mathrm{EO}_{8}\right)$ was purchased from Nikko Chemicals. 1,2,3trioctanoylglycerol (tricaplyrin, TOG) was purchased from Sigma-Aldrich Chemicals. 1,2,3-trihexanoylglycerol (tricaproin, THG) and 1,2,3-tridecanoylglycerol (tricaprin, TDG) were purchased from Tokyo Kasei Kogyo. These chemicals were used as received. Millipore filtered water was used to prepare the samples. Densities of $\mathrm{C}_{12} \mathrm{EO}_{8}\left(1.01 \mathrm{~g} \mathrm{~cm}^{-3}\right)$ and TOG (0.950 g $\mathrm{cm}^{-3}$ ) were measured by the oscillating U-tube density meter (Anton Paar, DSA-5000).

\subsection{Determination of Phase Diagrams}

The samples were prepared individually by weighing the appropriate amounts of the components in glass ampoules. The ampoules were flame-sealed immediately and the sample mixtures were homogenized using a vortex mixer. Viscous samples were homogenized further by repeated centrifugation in both directions through narrow constriction. The ampoules were kept in a temperature-controlled bath at $25^{\circ} \mathrm{C}$ for several minutes or days to bring the system at equilibrium. Phase identification was done by direct visual inspection and observing the samples through cross polarizers.

\section{3 Small-Angle X-ray Scattering (SAXS)}

SAXS measurements were performed on a smallangle scattering camera equipped with rotating anode and a CCD 2-D detector (Rigaku, NanoViewer). Ni-filtered $\mathrm{Cu}-\mathrm{K}_{\alpha}$ radiation $(\lambda=1.541 \AA)$ was used. The applied voltage and filament current were $40 \mathrm{kV}$ and 20 $\mathrm{mA}$, respectively. The samples of liquid crystals were filled in a hole of an iron plate and covered by plastic (Mylar) films. Sample thickness was kept at $1 \mathrm{~mm}$. The 
interlayer spacing, $d$, of the liquid crystals was obtained from the first Bragg peak.

Radius of lipophilic core of spherical micelle, $r_{\mathrm{I}}$, and a cross sectional area per surfactant molecule at the hydrophobic interface, $a_{\mathrm{S}}$, for a discontinuous cubic phase are evaluated by the following eqs. (1) and (2).

$$
\begin{aligned}
& r_{\mathrm{I}}=d \cdot\left(\frac{3}{4 \pi n_{\mathrm{C}}} \cdot\left(\phi_{\mathrm{L}}+\phi_{\mathrm{O}}\right)\right)^{1 / 3} \cdot C \\
& a_{\mathrm{S}}=\frac{3 v_{\mathrm{L}}}{r_{\mathrm{I}}} \cdot \frac{\phi_{\mathrm{L}}+\phi_{\mathrm{O}}}{\phi_{\mathrm{L}}}
\end{aligned}
$$

$\phi_{\mathrm{L}}$ and $\phi_{\mathrm{O}}$ are the volume fractions of lipophilic part of surfactant and oil in a system, respectively. $v_{\mathrm{L}}$ is the volume of lipophilic part of surfactant molecule, which is obtained by a summation of methyl and methylene unit volume (7). $n_{\mathrm{C}}$ is number of micelles in a unit cell of cubic phase. $C$ is a constant. For a simple cubic structure, $n_{\mathrm{C}}=1$ and $C=1$; a body-centered cubic structure, $n_{\mathrm{C}}=2$ and $C=\sqrt{2}$; for a face-centered cubic structure, $n_{\mathrm{C}}=4$ and $C=\sqrt{3}$.

Radius of lipophilic core of cylindrical micelle, $r_{\mathrm{H}}$, and $a_{\mathrm{S}}$ for a hexagonal liquid crystalline phase are evaluated by the following eqs. (3) and (4).

$$
\begin{aligned}
& r_{\mathrm{H}}=\left\{\frac{2}{\sqrt{3} \pi}\left(\phi_{\mathrm{L}}+\phi_{\mathrm{O}}\right)\right\}^{\frac{1}{2}} \cdot d \\
& a_{\mathrm{S}}=\frac{2 v_{\mathrm{L}}}{r_{\mathrm{H}}} \cdot \frac{\phi_{\mathrm{L}}+\phi_{\mathrm{O}}}{\phi_{\mathrm{L}}}
\end{aligned}
$$

Half thickness of lipophilic part in a bilayer, $d_{\mathrm{L}}$, and $a_{\mathrm{S}}$, for a lamellar liquid crystalline phase are evaluated by the following eqs. (5) and (6).

$$
\begin{aligned}
& d_{\mathrm{L}}=\frac{d}{2}\left(\phi_{\mathrm{L}}+\phi_{\mathrm{O}}\right) \\
& a_{\mathrm{S}}=\frac{v_{\mathrm{L}}}{d_{\mathrm{L}}} \cdot \frac{\phi_{\mathrm{L}}+\phi_{\mathrm{O}}}{\phi_{\mathrm{L}}}
\end{aligned}
$$

\section{Results and Discussion}

\section{$3 \cdot 1$ Effect of Solubilization of Oil on Cloud Temperature}

Cloud temperatures were measured by visual inspection for $2 \mathrm{wt} \%$ of aqueous solutions of $\mathrm{C}_{12} \mathrm{EO}_{8}$ with solubilized triglyceride oils. In Fig. 1, cloud temperatures are plotted against the oil content.

Cloud temperature of the aqueous $\mathrm{C}_{12} \mathrm{EO}_{8}$ solution without oil is $78^{\circ} \mathrm{C}$. Cloud temperature decreases by adding $0.25 \mathrm{wt} \%$ of triglyceride oils and it is almost unchanged with more addition of oil. Comparing three triglyceride oils, the largest decease of cloud temperature at the early stage of solubilization of oil is for the system with THG. To compare with other oils, we also show the results from a literature (13). Saturated hydrocarbons which tend to be solubilized in the core of micelles does not affect cloud temperature so much whereas SLIP, m-xylene and 1-dodecanol which tend to penetrate into surfactant palisade layer decrease cloud temperature very much. Present three triglyceride oils show similar trend as for the saturated hydrocarbons, suggesting that these triglyceride oils tend to be solubilized in the core of micelles. Among the system with saturated hydrocarbons, cloud temperature is lower with a hydrocarbon having smaller molecular weight. The cloud temperature of the system with these triglyceride oils is also the highest for TDG having the largest molecular weight and the lowest for THG having the smallest molecular weight among three triglyceride oils.

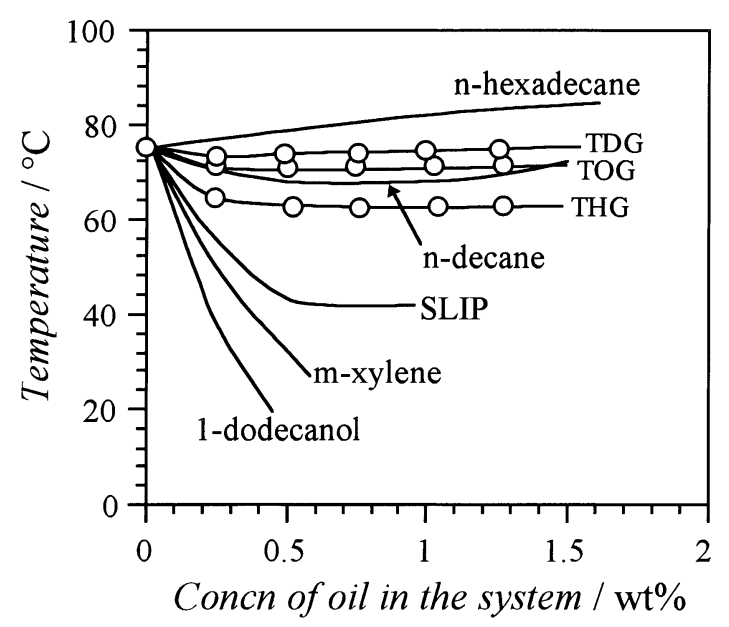

Fig. 1 Cloud Temperatures of $2 \mathrm{wt} \%$ Aqueous Solution of $\mathrm{C}_{12} \mathrm{EO}_{8}$ with Solubilized Triglyceride Oils are Plotted against Oil Content. Cloud temperatures with other oils taken from a literature (13) are also plotted as a reference. SLIP indicates sarcosinatelauroyl isopropyl. 


\section{$3 \cdot 2$ Phase Behavior in a Water/ $\mathrm{C}_{12} \mathrm{EO}_{8} / \mathrm{TOG}$ System}

A phase diagram in the water/ $\mathrm{C}_{12} \mathrm{EO}_{8} / \mathrm{TOG}$ system at $25^{\circ} \mathrm{C}$ is constructed and it is shown in Fig. 2.

On the water- $\mathrm{C}_{12} \mathrm{EO}_{8}$ axis, a micellar $\left(\mathrm{W}_{\mathrm{m}}\right)$, a hexagonal $\left(\mathrm{H}_{1}\right)$, a bicontinuous cubic $\left(\mathrm{V}_{1}\right)$ and a lamellar $\left(\mathrm{L}_{\alpha}\right)$ phase are successively formed with the increase in the surfactant concentration. As observed in various hydrophilic nonionic and ionic surfactant systems (15) the surfactant layer curvature of the binary water $/ \mathrm{C}_{12} \mathrm{EO}_{8}$ system is changed from highly positive to zero curvature. Note that a positive curvature is defined as a curvature which is convex toward water. When TOG is added to the aqueous micellar solution of $\mathrm{C}_{12} \mathrm{EO}_{8}$, it is solubilized in the micelle. However the amount of oil solubilized in the micellar solution is not very large, i.e. solubilized oil is less than $1 \mathrm{wt} \%$ at 10 wt $\%$ surfactant in water, less than $2 \mathrm{wt} \%$ at $20 \mathrm{wt} \%$ surfactant in water. Since $\mathrm{C}_{12} \mathrm{EO}_{8}$ is rather hydrophilic surfactant and its spontaneous curvature is large, it is not allowed forming large spherical micelles containing a

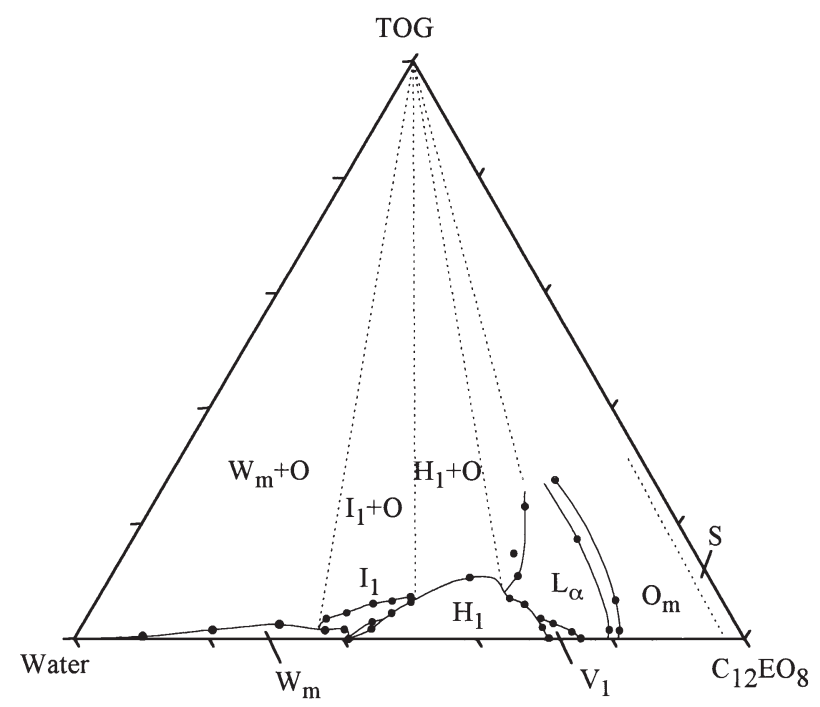

Fig. 2 Phase Diagram of Water $/ \mathrm{C}_{12} \mathrm{EO}_{8} / \mathrm{TOG}$ System at $25^{\circ} \mathrm{C} . \mathrm{W}_{\mathrm{m}}, \mathrm{I}_{1}, \mathrm{H}_{1}, \mathrm{~V}_{1}, \mathrm{~L}_{\alpha}, \mathrm{O}_{\mathrm{m}}$ and $\mathrm{S}$ indicate a micellar phase, a discontinuous cubic phase, a hexagonal phase, a bicontinuous cubic phase, a lamellar phase, a reverse micellar phase and a solid phase, respectively. $\mathrm{W}_{\mathrm{m}}+\mathrm{O}, \mathrm{I}_{1}+\mathrm{O}$ and $\mathrm{H}_{1}+\mathrm{O}$ indicate a two-phase equilibrium of $\mathrm{W}_{\mathrm{m}}, \mathrm{I}_{1}$ and $\mathrm{H}_{1}$ with an excess oil phase, respectively. The $\mathrm{I}_{1}$ phase is formed as an island, which is separated from the water-surfactant axis. large amount of solubilized oil. Oil-solubilization capacity in micelles and liquid crystals increases with the surfactant concentration since the curvature of surfactant layer becomes less positive and it allows forming large surfactant aggregates containing a large amount of oil.

If we see the change in self-assembled structures by adding TOG, $\mathrm{H}_{1}-\mathrm{I}_{1}$ transition on the left hand side of the hexagonal region and $\mathrm{H}_{1}-\mathrm{L}_{\alpha}$ transition at right hand side is observed. The surfactant layer curvature is changed to more positive in the $\mathrm{H}_{1}-\mathrm{I}_{1}$ transition and to less positive in the $\mathrm{H}_{1}-\mathrm{L}_{\alpha}$ transition. The water molecules hydrated to the poly(oxyethylene) chain decreases with the increase in the $\mathrm{C}_{12} \mathrm{EO}_{8}$ concentration in water (16). Consequently, the effective volume of the hydrophilic chain of $\mathrm{C}_{12} \mathrm{EO}_{8}$ decreases and also the repulsion between neighboring hydrophilic chains decreases, which reduces the highly positive curvature. Hence the spontaneous curvature of the surfactant layer in the hexagonal phase is high at relatively low surfactant concentration and low at high concentration. It is reported $(1,13,17,18)$ that the surfactant layer curvature is changed to opposite direction by adding oil depending on the surfactant hydrophilicity or the spontaneous curvature of surfactant layer, in which the highly positive curvature is changed to more positive by the solubilization of oil but the less positive curvature decreases more or it is changed to a negative curvature. Therefore the $\mathrm{H}_{1}-\mathrm{I}_{1}$ transition at low surfactant concentration and the $\mathrm{H}_{1}-\mathrm{L}_{\alpha}$ transition at high surfactant concentration are observed by adding TOG.

\subsection{Changes in Microstructure of Liquid Crystals by Adding Oil}

SAXS measurement was performed to study the structural change in the liquid crystals by adding oil. An interlayer spacing, $d$, was obtained from a Bragg peak of a SAXS spectrum. The results are shown in Fig. 3 for the $\mathrm{I}_{1}, \mathrm{H}_{1}$ and $\mathrm{L}_{\alpha}$ phases.

$r_{\mathrm{I}}, r_{\mathrm{H}}, d_{\mathrm{L}}$ and $a_{\mathrm{S}}$ are calculated from eqs. (1)-(6) and also plotted in Fig. 3. For the $I_{1}$ phase, we used the constants of a face-centered structure for eqs. (1) and (2). For all single-phase regions of liquid crystals, $d, r_{\mathrm{I}}, r_{\mathrm{H}}$ and $d_{\mathrm{L}}$ increase as the oil content increases. $a_{\mathrm{S}}$ decreases for the $\mathrm{I}_{1}$ phase and it is almost constant for the $\mathrm{H}_{1}$ and $\mathrm{L}_{\alpha}$ phases.

When oil molecules are solubilized in surfactant aggregates, these are partitioned in two sites in the inte- 
(a) water $/ \mathrm{C}_{12} \mathrm{EO}_{8}=60 / 40$

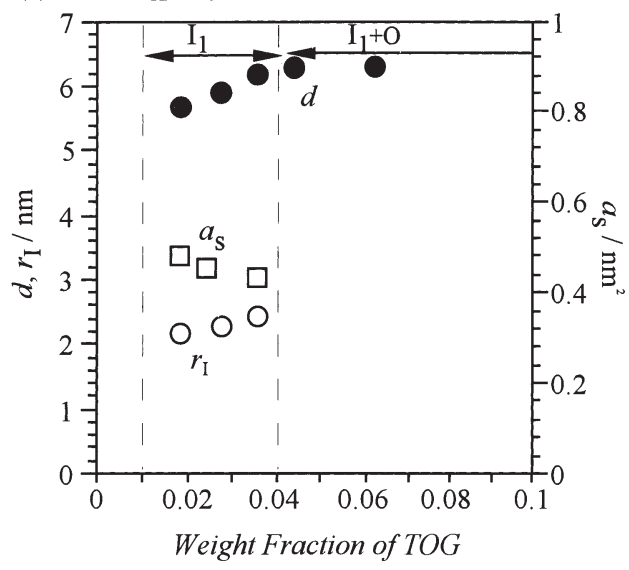

(b) water $/ \mathrm{C}_{12} \mathrm{EO}_{8}=40 / 60$

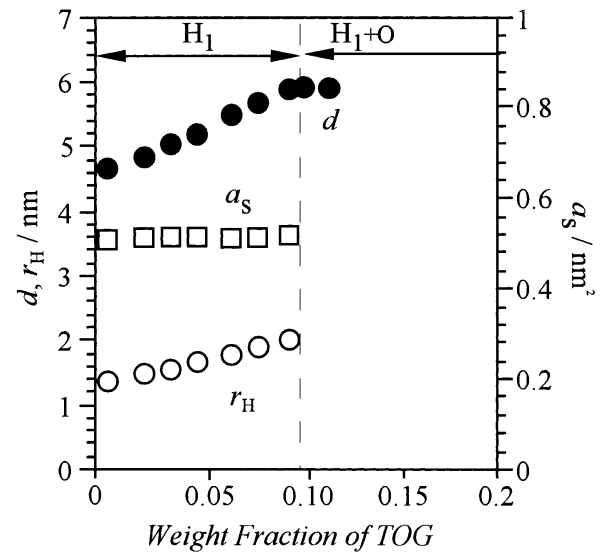

(c) water $/ \mathrm{C}_{12} \mathrm{EO}_{8}=28 / 72$

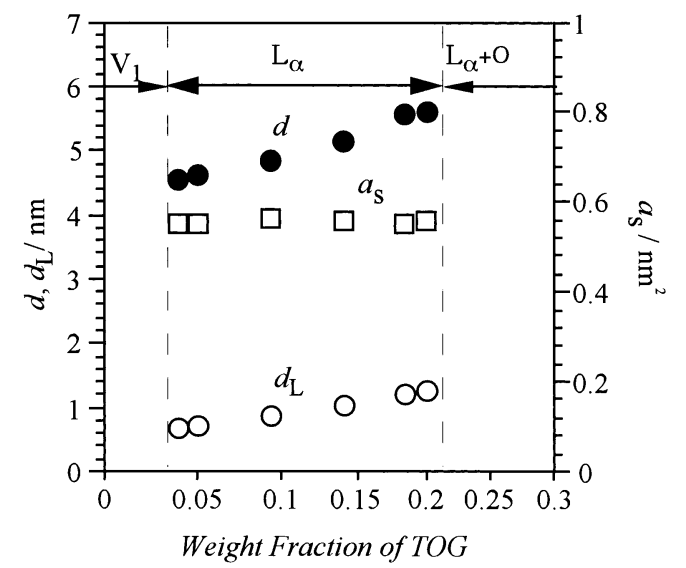

Fig. 3 Interlayer Spacing, $d$, for $\mathrm{I}_{1}, \mathrm{H}_{1}$ and $\mathrm{L}_{\alpha}$ Phases is Plotted against the Weight Fraction of TOG. Effective cross sectional area per surfactant molecule at hydrophile-lipophile interface, $a_{\mathrm{S}}$, radius of lipophilic core of spherical micelle in $\mathrm{I}_{1}$ phase, $r_{\mathrm{I}}$, radius of lipophilic core of cylindrical micelle in $\mathrm{H}_{1}$ phase, $r_{\mathrm{H}}$ and half thickness of lipophilic bilayer in $\mathrm{L}_{\alpha}$ phase, $d_{\mathrm{L}}$, are also plotted. The water $/ \mathrm{C}_{12} \mathrm{EO}_{8}$ ratio is fixed at (a) 6/4 for $\mathrm{I}_{1}$ phase, (b) 4/6 for $\mathrm{H}_{1}$ phase and (c) 28/72 for $\mathrm{L}_{\alpha}$ phase.

rior of surfactant aggregates, i.e. core and palisade layer. In the case of solubilization in core, it is reported that $a_{\mathrm{S}}$ is almost constant for $\mathrm{H}_{1}$ and $\mathrm{L}_{\alpha}$ phases (1) and decreases for $I_{1}$ phase (12), which appear as same trend as in Fig. 3. Increasing $r_{\mathrm{I}}, r_{\mathrm{H}}$ and $d_{\mathrm{L}}$ also indicates the oil solubilization in core (1). Hence TOG molecules in the present system are almost solubilized in the core of aggregates.

\subsection{Effect of Temperature and Oil Molecu- lar Size on the Cubic Phase}

Figure 4 is a phase diagram as functions of weight fraction of TOG and temperature at constant water-to$\mathrm{C}_{12} \mathrm{EO}_{8}$ ratio (56/44).
An $\mathrm{H}_{1}$ phase is formed in the oil-free system and the $\mathrm{H}_{1}$ phase is changed into an $\mathrm{I}_{1}$ phase by adding a small amount of oil. The melting temperature of the $I_{1}$ phase is about $30^{\circ} \mathrm{C}$ with $3 \%$ of oil and it abruptly increases by increasing oil content and reaches up to $43^{\circ} \mathrm{C}$. In other words, the cubic phase is stabilized against temperature by solubilizing oil. Similar thermal-stabilization effect for $\mathrm{I}_{1}$ phase is also observed in other surfactant systems $(4,5)$.

The maximum oil content in the cubic phase and the micellar phase increases by increasing temperature. The spontaneous curvature of surfactant layer decreases by increasing temperature for poly(oxyethylene)-type nonionic surfactant due to the dehydration from 


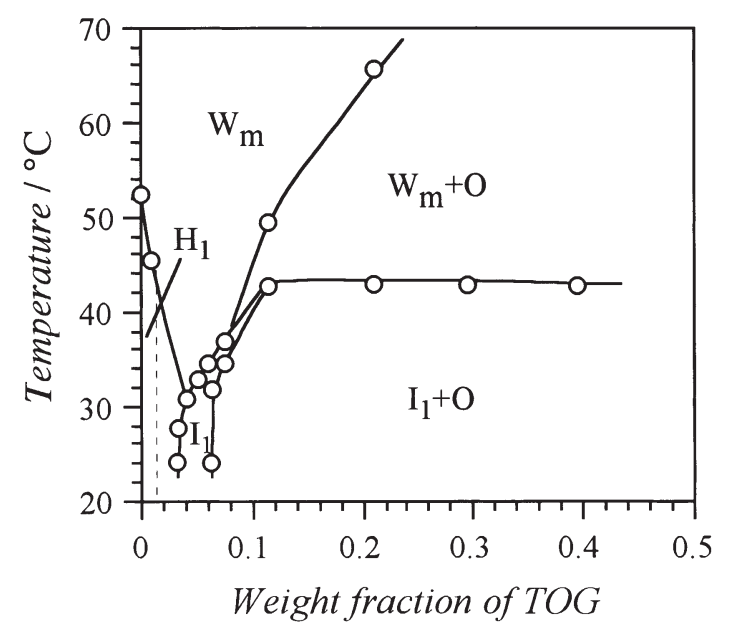

Fig. 4 Phase Diagram of Water/ $\mathrm{C}_{12} \mathrm{EO}_{8} / \mathrm{TOG}$ System at Fixed Water/ $\mathrm{C}_{12} \mathrm{EO}_{8}$ Ratio, 56/44. Weight fraction of TOG and temperature are variables. An $\mathrm{H}_{1}-\mathrm{I}_{1}$ phase transition takes place by adding small amount of TOG to the aqueous $\mathrm{C}_{12} \mathrm{EO}_{8}$ system. The melting temperature of the $I_{1}$ phase increases with increasing oil content.

poly(oxyethylene) chain (19). Therefore larger micelles can be formed at higher temperature and the larger micelles can solubilize more oil.

Figure 5 shows the position of $\mathrm{I}_{1}$ phases in the water $/ \mathrm{C}_{12} \mathrm{EO}_{8} /$ oil systems.

Three different oils are used, i.e. THG, TOG, TDG. The $\mathrm{I}_{1}$ phases for the systems with TOG and TDG appear in similar composition range whereas the $\mathrm{I}_{1}$ phase for the THG system shifts to higher oil content. It is reported that the cubic phase shifts to higher oil content with oil having smaller molecular weight (20) since the amount of oil molecules that penetrate into the surfactant palisade layer in micelles increases, which allows forming larger micelles having smaller surfactant layer curvature. Although these triglyceride oils are mostly solubilized in the core of micelles, oil with smaller molecular weight tends to penetrate more, which causes the difference in the cloud temperature as is shown in Fig. 1. The molecular weight of THG molecule is smaller than the other two oils. Therefore the $I_{1}$ phase for the THG system is formed with higher oil content. However the $I_{1}$ phases of the TOG and TDG system is not so different although the molecular weight is different. The $I_{1}$ phase for the TOG system is very close to the aqueous surfactant system (bottom axis).

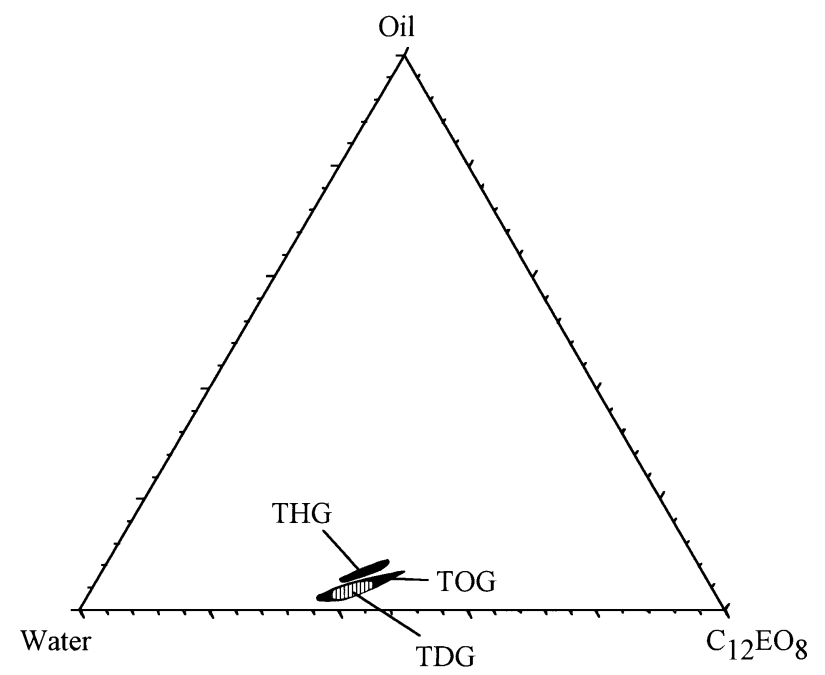

Fig. 5 Cubic Phase Regions for Water $/ \mathrm{C}_{12} \mathrm{EO}_{8} /$ Triglyceride Oil Systems at $25^{\circ} \mathrm{C}$ are Mapped in a Triangle Composition Diagram. The cubic phase region for the TDG system (shaded area) is included in the cubic phase region for the TOG system.

Therefore, even if the penetration tendency of TOG molecules is higher than the TDG molecules, the $\mathrm{I}_{1}$ phase cannot shift to lower oil content. In the case of the TDG system, the region of the $I_{1}$ phase is shrunk instead of shifting the region.

\section{Conclusion}

The phase behavior and the microstructures of liquid crystals were studied in water $/ \mathrm{C}_{12} \mathrm{EO}_{8} /$ triglyceride oil systems. From the changes in cloud temperatures and effective cross sectional area of surfactant molecule in liquid crystals with varying the oil content, triglyceride oils in this study (1,2,3-trihexanoylglycerol, 1,2,3-trioctanoylglycerol and 1,2,3-tridecanoylglycerol) is mainly solubilized in the core of surfactant self-assemblies, which is similar solubilization behavior as saturated hydrocarbons. Therefore the micellar phase and the hexagonal phase turn into the discontinuous cubic phase by adding these triglyceride oils. The needed amount of oil solubilization to form the discontinuous cubic phase is smaller in the case of triglyceride oil with smaller molecular weight. It was also found that the discontinuous cubic phase stabilized against temperature by the solubilization of oil. 


\section{Acknowledgements}

This work was supported by The Ministry of Education, Culture, Sports, Science and Technology, Grantin-Aid for Young Scientists (B), No.15780098.

\section{References}

1. H. KUNIEDA, K.OZAWA and K.-L. HUANG, Effect of Oil on the Surfactant Molecular Curvatures in Liquid Crystals, J. Phys. Chem., Vol. 102, 831-838 (1998).

2. T. SUZUKI, Secondary Droplets Formed in O/W Emulsions, Yukagaku, Vol. 35, 113-119 (1986).

3. Y. SUZUKI and H. TSUTSUMI, Emulsifying Characteristics of $\alpha$-Monoalkyl Glyceryl Ether, Yukagaku, Vol. 36, 588-593 (1987).

4. C. ROdRigueZ, K. ShigetA and H. KUNIEDA, CubicPhase-Based Concentrated Emulsions, J. Colloid Interface Sci., Vol. 223, 197-204 (2000).

5. H. KUNIEDA, K. ARAMAKI, T. NISHIMURA and M. ISHITOBI, Phase Behavior of Polyglycerin Fatty Acid Ester in a Water-Oil System and Formulations of Gel-Emulsions Stabilized by the Cubic Phase, J. Jpn Oil Chem. Soc., Vol. 49, 617-624 (2000).

6. Md.H. UDDIN, C. RODRIGUEZ, K. WATANABE, A. LOPEZQUINTELA, T. KATO, H. FURUKAWA, A. HARASHIMA and H. KUNIEDA, Phase Behavior and Formation of Reverse Cubic Phase Based Emulsion in Water/Poly(oxyethylene) Poly (dimethylsiloxane) Surfactants/Silicone Oil Systems, Langmuir, Vol. 17, 5169-5175 (2001).

7. H. KUNIEDA, K. SHIGETA, K. OZAWA and M. SUZUKI, Self-Organizing Structures in Poly(oxyethylene) Oleyl EtherWater System, J. Phys. Chem. B, Vol. 101, $7952-7957$ (1997).

8. Md.H. KHALID, S. HINATA, A. LOPEZ-QUINTELA and H. KUNIEDA, Phase Behavior of Poly(oxyethylene)Poly(oxypropylene)-Poly(oxyethylene) Block Copolymer in Water and Water- $\mathrm{C}_{12} \mathrm{EO}_{5}$ Systems, J. Dispers. Sci. Technol., Vol.
24, 411-422 (2003).

9. M. ISHITOBI and H. KUNIEDA, Effect of Distribution of Hydrophilic Chain on the Phase Behavior of Polyglycerol Fatty Acid Ester in Water, Colloid Polym. Sci., Vol. 278, 899-904 (2000).

10. N. KANEI, T. IMAE and H. KUNIEDA, Phase Behavior in Water-Sucrose Monoalkanoate Systems, J. Jpn Oil Chem. Soc., Vol. 45, 849-855 (1996).

11. K. ARAMAKI and H. KUNIEDA, Solubilization of Oil in Mixed Cationic Surfactant Liquid Crystal, Colloid Polym. Sci., Vol. 277, 34-40 (1999).

12. K. ARAMAKI, Md.H. KABIR, N. NAKAMURA, H. KUNIEDA and M. ISHITOBI, Formation of Cubic-Phase Microemulsions in Sucrose Alkanoate Systems, Colloid Surf. A, Vol. 183185, 371-379 (2001).

13. H. KUNIEDA, M. HORII, M. KOYAMA and K. SAKAMOTO, Solubilization of Polar Oils in Surfactant Self-organized Structures, J. Colloid Interface Sci., Vol. 236, 78-84 (2001).

14. H. HOFFMANN and W. ULBRICHT, Transition of Rodlike to Globular Micelles by the Solubilization of Additives, J. Colloid Interface Sci., Vol. 129, 388-405 (1989).

15. R.G. LAUGHLIN, The Aqueous Phase Behavior of Surfactant, Academic Press, San Diego, pp. 111-307 (1994).

16. P.G. NILSSON and B. LINDMAN, Water Self-Diffusion in Nonionic Surfactant Solutions. Hydration and Obstruction Effects, J. Phys. Chem., Vol. 87, 4756-4761 (1983).

17. H. KUNIEDA, K. SHIGETA and M. SUZUKI, Phase Behavior and Formation of Reverse Vesicles in Long-PolyoxyethyleneChain Nonionic Surfactant Systems, Langmuir, Vol. 15, 31183122 (1999).

18. H. KUNIEDA, G. UMIZU and K. ARAMAKI, Effect of Mixing Oils on the Hexagonal Liquid Crystalline Structures, J. Phys. Chem. B, Vol. 104, 2005-2011 (2000).

19. R. STREY, Microemulsion Microstructure and Interfacial Curvature, Colloid Polym. Sci., Vol. 272, 1005-1019 (1994).

20. Md.H. KABIR, K. ARAMAKI, M. ISHITOBI and H. KUNIEDA, Cloud Point and Formation of Microemulsions in Sucrose Dodecanoate Systems, Colloid Surf. A, Vol. 216, 65-74 (2003). 